\title{
INNOVACIONES EN LA GESTIÓN DEL DESARROLLO LOCAL: LA EXPERIENCIA DE PORTO ALEGRE**
}

\author{
Suzana Moura**
}

Fecha de recepción: febrero de 1999

Fecha de aceptación y versión final: agosto de 1999

Resumen: El presente artículo versa sobre las perspectivas de gestión del desarrollo local en el municipio de Porto Alegre en Brasil, donde se vienen desarrollando estrategias de democratización de carácter innovador en lo que se refiere a las relaciones entre el gobierno y la sociedad civil. El análisis crítico del discurso y de algunas de las iniciativas tomadas por el gobierno que se hace en este trabajo ponen en evidencia las posibilidades y los límites de la acción local en materia de desarrollo económico. Con base en la literatura existente, se discute el activismo democrático y el estilo de gerencia aquí denominada "emprendedurismo" competitivo como tendencias innovadoras en la gestión local de los 90 . A través de algunas datos empíricos, se discuten las bases de estrategia de desarrollo local del municipio y los factores que potencian y limitan el proceso.

Palabras clave: Desarrollo local, participación ciudadana, gestión municipal, innovaciones democráticas, emprendedurismo competitivo.

Abstract: This paper deals with the prospects of local development management in the Municipality of Porto Alegre, Brazil. This city's council has been developing new strategies of democratization in relation to the articulation between the government and civil society. The critical analysis of the discourse and of some of the government's proposals evidences the possibilities and limits of local actions related to economic development. Based upon the current literature on this topic, the article debates the democratic activism and the competitive enterprise as tendencies of innovation in the local management of the 90 s. Empirical information about the Municipality of Porto Alegre and the bases of its local development strategy are given in order to discuss the factors that facilitate or difficult the process.

Key words: Local development, citizen's participation, municipal decision-making process, democratic innovations and competitive management.

\section{INTRODUCCIÓN}

La (re)valorización de los gobiernos locales es un tema que está presente en la agenda del debate del medio académico y entre los actores sociales desde los 80 . Uno de los elementos impulsores fue la sucesión de reformas de descentralización del Estado desarrolladas en Europa -en especial Italia, Francia y España- y, también, en América Latina: Chile, Colombia, Perú, Bolivia y Brasil, entre otros países.

En Brasil se dio un proceso de ampliación de la autonomía municipal en la transición democrática de los 80 que culminó con la elaboración de la Constitución de 1988. Anteriormente, el municipio ya disponía de capacidad legislativa propia, autonomía para elegir directamente a los miembros del legislativo (concejales) y al jefe del ejecutivo (alcalde), y competencia para recaudar tributos (impuestos, tasas y contribución de mejorías), además de compartir los impuestos recaudados por otras esferas de gobierno. Pero durante el régimen militar esas autonomías estaban limitadas y no había elecciones directas para alcalde de las capitales y áreas consideradas estratégicas por el gobierno. La Constitución de 1988 avanzó en cuanto a la definición del municipio como miembro de la federación, el aumento del número de impuestos municipales y el incremento de las transferencias de los Estados y el Gobierno Central a los municipios.

En la medida en que las reformas estuvieron asociadas a las transiciones democráticas o al avance del neoliberalismo, prevalecieron dos vertientes de análisis. Se enfatizaron, por un lado, las virtudes de lo local en materia de desarrollo y/o del ejercicio de la democracia; por otro, los efectos perversos y la funcionalidad de la descentralización.

* Este trabajo es fruto de una investigación realizada para la tesis de doctorado "Ciudades Emprendedoras, Ciudades Democráticas y Redes Públicas", que fue finalizada en abril de 1997 dentro del Núcleo de Posgraduación en Administración de la Escuela de Administración de la Universidad Federal de Bahia. Incorpora también datos de una investigación más reciente, "La Gestión del Desarrollo Local y las Posibilidades de Financiación", que cuenta con el apoyo financiero del Consejo Nacional de Desarrollo Científico y Tecnológico. El trabajo está integrado en el proyecto "Ciudades Estratégicas, Organizaciones y Desarrollo Local", del Núcleo de Estudios sobre Poder y Organizaciones Locales (NEPOL) del Núcleo de Posgraduación en Administración (NPGA) de la Escuela de Administración de la Universidad Federal de Bahia (EAUFBA).

*** Profesora e investigadora de la Escuela de Administración de la UFBA, e integrante del NEPOL/NPGA/EAUFBA.凶 suzmoura@ufba.br 
Este tipo de polarización puede superarse cuando se tiene en cuenta la diversidad de situaciones que devienen tanto de los procesos de descentralización como de las respuestas tejidas en la esfera local en razón de diversos factores, bien sean objetivos o subjetivos, macroestructurales o locales. Se evidencia lo local como propulsor de iniciativas innovadoras y se abre un importante campo de investigación sobre lo que se está produciendo de nuevo en las formas de gestión pública, en un contexto caracterizado por la crisis y las reestructuraciones del Estado y de la economía.

En esa línea de aproximación se encuentran estudios que evidencian, por lo menos, dos tendencias de innovación. Una que se afirma a partir de los 80 , aquí denominada "emprendedurismo competitivo", y otra, ya presente en los 70, que se puede denominar "activismo democrático".

La primera indica un movimiento de redefinición en el papel y actuación de los gobiernos locales, con énfasis en el desarrollo de ventajas comparativas y en la búsqueda de una mayor eficiencia en la gestión urbana, persiguiendo la integración competitiva en el mercado global. Se destacan otros elementos característicos que aparecen como innovación: el gobierno asume un papel de catalizador/articulador de fuerzas; se privilegia la construcción de espacios y mecanismos de cooperación público-privado, así como la formación de consensos en torno a proyectos estratégicos; finalmente, gana relevancia la utilización de prácticas de gerencia empresarial en la gestión local y el "marketing" urbano para la promoción externa e interna de la ciudad. Tal definición agrega contribuciones de varios autores que tienen como referencias los procesos de ciudades americanas y europeas a partir de la década de los 80; entre ellos, se destacan: Harvey (1989) -cuando discute el nuevo "emprendedurismo" urbano-; Borja (1994) -cuando trata del "protagonismo citadino" - y Osborne y Gaebler (1993) -en su abordaje sobre el "gobierno emprendedor"-'.

Este fenómeno es visualizado y analizado en América Latina más recientemente. Borja (1994) observa que los procesos de descentralización y democratización de los años 80 , aunque hayan conducido a una revalorización de los gobiernos locales, no implicaron, de inmediato, un avance en relación al "protagonismo", que sólo aconteció a partir de los 90 a través de iniciativas de implementación de planes estratégicos, como sucedió en Río de Janeiro, Bogotá y Córdoba ${ }^{2}$. En Brasil existen algunos estudios que, a partir de las referencias de Harvey (1989), analizan determinadas experiencias locales en cuanto expresiones de gerenciamiento o "emprendedurismo" urbano, como las de Curitiba (Pinahez, 1993 y Silva, 1993) y del consorcio Municipal del ABC paulista, iniciado en 1991 (Pacheco, 1993).

Mientras que el "emprendedurismo" competitivo ha sido poco analizado, el activismo democrático adquirió una importante visibilidad en Brasil en materia de estudios. Se refiere a un conjunto de ideas y prácticas de gestión local defendido por gobiernos de izquierda y progresistas, que enfatiza el aspecto de extensión -o radicalización- de la democracia y la ciudadanía, en términos políticos y económicos. Como elementos demarcadores se distinguen: la búsqueda de construcción de espacios de participación popular en la gestión local, redefinidos, más recientemente, como nuevas esferas públicas de ejercicio de la ciudadanía activa y de control sobre el Estado; el direccionamiento para las demandas sociales mediante la inversión de prioridades de las políticas municipales; la democratización de las informaciones y el acceso a los bienes y servicios públicos; y la construcción de una nueva cultura política. Entre los autores que analizan esa tendencia, se citan: Leal (1994), Daniel (1990 y 1994), Moura (1993 y 1994), Kowarik y Singer (1993), Caccia Bava (1994) y Pacheco (1993).

El hecho de que el "emprendedurismo" haya sido reforzado en los 90, al mismo tiempo en que disminuían las demandas en torno a las cuestiones democráticas pudiera ser interpretado como que estas últimas fueron substituidas por la agenda de la competitividad y la eficiencia. Se confronta este tipo de interpretación con la discusión actual del activismo democrático en Brasil y con la posibilidad de renovación e incorporación de trazos de "emprendurismo". Se discute que tal renovación devenga, entre otros factores, del empeoramiento de la problemática del desempleo que ha obligado a los gobiernos locales a buscar alternativas de creación de empleo y renta y de desarrollo local; discusión que aquí es desarrollada en base a la experiencia de Porto Alegre.

\section{CAMINOS DE INNOVACIÓN}

Porto Alegre es la capital del estado de Río Grande del Sur, posee una población aproximada de 1.300 .000 habitantes, siendo el núcleo de una región metropolitana cuya población es de 3 millones de habitantes.

Desde 1989, el municipio viene siendo gobernado por un frente de partidos de izquierda ${ }^{3}$-denominado Frente Popular- destacándose el Partido de los Trabajadores (PT) que, desde entonces, asumió la dirección del ayuntamiento indicando al alcalde y vicealcalde en la siguiente secuencia: Olívio Dutra y Tarso Genro (89/92); Tarso Genro y Raul Pont (93/96); y Raul Pont y José Fortunati (97/2000). Obsérvase, pues, un hecho poco común en la realidad brasileña: la continuidad de gobierno. Inclusive el vice de cada gestión es alcalde en la siguiente, lo que ha permitido el perfeccionamiento de algunas experiencias y, a la vez, producido ideas que se diferencian en el tiempo.

Inicialmente, el gobierno focalizó su actuación en el mejoramiento de los servicios municipales, fundamentalmente aquellos relacionados con los sectores y áreas populares, como el transporte colectivo, saneamiento básico, educación, salud y pavimentación de calles en los barrios; siempre siguiendo una orientación de inversión de prioridades que integra un repertorio del ideario democrático de la gestión local. Ello desencadenó un proceso de democra-

1. Además de éstos, otros autores, como LE GALÉs (1994, 1995), Biarez y Nivers (1994) y BATLEy (1991), abordan el mismo fenómeno.

2. Esas y otras ciudades adoptaron el modelo de planificación estratégica de Barcelona, del que Borja fue uno de los colaboradores y difusores.

3. Además del PT, el Frente Popular estaba compuesto en 1989 por el Partido Socialista Brasileño (PSB) -que se retiró en las elecciones de 1996- y por el Partido Comunista Brasileño (PCB), que en 1990 pasó por un proceso de reformulación, resultando en otro partido con la sigla PPS (Partido Popular Socialista). 
tización de las decisiones, siendo el Presupuesto Participativo ${ }^{4}(\mathrm{PP})$ uno de los ejes centrales.

En 1991 se tomaron otras iniciativas que ampliaron las acciones existentes, con el objeto de enfrentar la crisis económica. El gobierno invitó a sindicatos, asociaciones comunitarias y empresariales para formar un Forum contra la Recesión y el Desempleo. A través del Forum se llevaron a cabo acciones en el área del abastecimiento popular para abaratar los alimentos, además de otras acciones dirigidas a la dinamización de la economía local tales como: la mejoría en la infraestructura de las áreas comerciales y de las ferias de artesanía y alimentos; el desarrollo de procedimientos para facilitar la licencia de actividades y el registro de micro y pequeñas empresas, entre otras. A partir de ahí se dio un cambio en la relación con los agentes económicos, que antes era más conflictiva.

Cabe aclarar que ya existía una secretaría de industria y comercio, pero su actuación, hasta entonces, se restringía básicamente a la licencia y fiscalización de las actividades económicas.

Adicionalmente, se implementaron varios proyectos económicos, particularmente en el segundo gobierno, que ampliaron el foco de actuación del ayuntamiento en dirección a la producción de nuevas tecnologías relacionada con el trabajo, a la promoción de la ciudad (Corredor Cultural de la Ciudad Antigua, Programa de Eventos Culturales, Ferias Promocionales, etc.), al fomento de Redes de Pequeños y Medios Emprendimientos y a la descentralización de las actividades económicas en la ciudad.

\section{UNA ESTRATEGIA DE DESARROLLO LOCAL}

La estrategia que el gobierno iba construyendo con ese conjunto de acciones en el campo del desarrollo económico era la de convertirse en un "gestor político de la economía local", o sea:

"(el poder público) tiene una visión sobre toda la actividad económica de la ciudad y propone un camino. Ese camino se propone, evidentemente, a partir de su programa político, de su visión del mundo, de su inserción social y, a partir de ahí, es un sujeto activo en la actividad económica [...] que tiene como objetivo hacer que el capital sea redistribuido [...] Una estrategia que propone una reorganización del modo de funcionar de la economía creando proyectos y un ambiente que posibilite el control de la comunidad sobre el mercado [...] ampliando la capacidad de la comunidad de planificar la actividad económica [...] organizando a los excluidos socialmente como una fuerza económica estratégica" (Milton Pantaleão, Subsecretario de Producción, Industria y Comercio, entrevistado el 24/04/97).

Como parte de esa estrategia, el gobierno se proponía actuar de modo articulado con los agentes interesados, constituyendo espacios públicos de los sectores populares a los empresariales, pasando por los sectores medios (universidades, asociaciones profesionales, etc.). Aquí el ideario inicial de la democratización y participación popular en la gestión municipal asume, también, un enfoque de negociación y de búsqueda de cooperación entre lo público y lo privado, entre el gobierno y la sociedad civil.

En este proceso, el propio Presupuesto Participativo se amplió en función del número de actores que articula, al agregar a las sesiones plenarias regionales, plenarias temáticas. Nótese que una de ellas se denomina justamente plenaria de Desarrollo Económico; otras plenarias son: de la Reforma y Desarrollo Urbano, Educación y Cultura, Circulación y Transporte y Financiamiento de la Ciudad. Tal cambio ocurrió a partir de 1993, facilitando la participación de actores sociales involucrados en proyectos económicos y que se mantenían distanciados del proceso de discusión pública sobre el presupuesto.

La preocupación del segundo gobierno fue, también, la de ampliar el horizonte de la discusión pública sobre la planificación municipal más de un año (el tiempo del plan presupuestario). De este modo, surgió en 1993 Ciudad Constituyente, proyecto que contempla la definición de directrices estratégicas de desarrollo que orienten las acciones municipales 5 . Si bien la experiencia tuvo sus altos y bajos y concluyó con cierto vacío político en 1996, ella merece ser analizada en la medida que refleja un camino de renovación del activismo democrático.

Tal renovación puede ser interpretada como una de las tendencias observadas en los años 90 , cuando el discurso y la práctica de la participación popular pasaron a contemplar al conjunto de la sociedad, inclusive a los agentes económicos, con una agenda caracterizada por la atención de las demandas populares más inmediatas y localizadas. Tal aspecto puede evidenciarse tanto en los objetivos de Ciudad Constituyente -la discusión pública de un "proyecto de futuro" y globalizante de la ciudad-, como en los procedimientos adoptados -los ejes temáticos ${ }^{6}$-, la llamada a las organizaciones empresariales y sectores medios y populares para integrar la Coordinación General y los Grupos de Trabajo, y la realización de eventos movilizadores con discusión de temas estratégicos.

En esta renovación, al mismo tiempo en que se reproducen ideas que reflejan aquel ideario, se producen otras reflejo de la tendencia aquí denominada "emprendedurismo" competitivo, aunque con matices diferenciados debido a la

4. Se trata de un proceso de discusión pública del presupuesto municipal, a partir del cual la población participa de la decisión sobre inversiones, acompaña la ejecución y obtiene informaciones. Tal proceso se desarrolla en base a plenarias temáticas, las cuales eligen representantes que componen un consejo municipal. Mayores informaciones sobre el proceso se encuentran en FEDOZZI (1997) y GIACOMONI (1993).

5. El desarrollo fue estructurado a partir de grupos temáticos (Desarrollo Económico, Desarrollo y Reforma Urbana, Circulación y Transporte y Financiamiento de la Ciudad), de una Coordinación General y otro espacio denominado Congreso de la Ciudad. En Ciudad Constituyente convergieron ideas y proyectos de gobierno y de actores de la socie dad civil, cubriendo con limitaciones el campo popular (consejeros del PP, Forum de Reforma Urbana, CUT...), sectores medios (UFRGS, IAB...) y empresariales (SINDUSCON, Asociación Comercial...). Para mayores informaciones sobre Ciudad Constituyente ver MOURA (1997).

6. Se organizó una discusión pública alrededor de los siguientes ejes temáticos, a partir de los cuales se formaron los grupos de trabajo: Desarrollo Económico, Reforma y Desarrollo Urbano, Financiamiento de la ciudad y Circulación y Transporte. 
hegemonía de un proyecto político que enfatiza la democratización radical del Estado y la sociedad. Proyecto que pretende convertirse en una referencia nacional para disputar las demás esferas del gobierno, como alternativa a lo que se considera el proyecto político hegemónico en la actualidad, el neoliberalismo.

Se incorporó la preocupación por la construcción de nuevas estrategias locales de desarrollo como algo que integra el repertorio del gobierno local en la actualidad. Esta preocupación aparece como uno de los ejes de discusión del proyecto. Al mismo tiempo, la óptica de la competitividad, según la lógica de mercado, está, en cierta medida, subordinada a la del ideario, conforme se observa en los análisis, directrices y propuestas definidas. A continuación se destacan algunos ejemplos ilustrativos:

- La idea de atracción y competitividad de la ciudad se vincula a una visión de desarrollo equilibrado que integra el objetivo de "combate a las desigualdades y a la exclusión social" y al fomento a la pequeña y media empresa, además de otros aspectos referentes a las cuestiones ambientales y de la cultura local.

- Las medidas adoptadas evidencian lo anterior: junto con las actividades de "implementar una comisión de la Tecnópolis de Porto Alegre", "crear facilidades de comercialización (por ejemplo el Trade Point)" y "Divulgar Porto Alegre y sus atracciones en el Cono Sur y en el país"; también se destacan la "Creación de Cunas Empresariales [...] para pequeños emprendedores" y la "Creación del sector de asesoramiento técnico [...] para comunidades de trabajo, producciones domésticas, micro y pequeñas empresas".

- La cuestión de la integración a los mercados, en este caso al Mercosur, se aborda procurando negar lo que sería la lógica dominante: la "estrategia de venta de la ciudad a bajo precio y a cualquier costo y, también, la competencia fiscal o del mercado entre ciudades y regiones" 7 .

Esa perspectiva de integración es, en cierta forma, puesta en práctica con el proceso de articulación que resultó en la formación de la Red Merco-Ciudad en 1995. En este proceso se destacan las ideas de integrar en las relaciones comerciales a "pequeños y medios emprendedores" y de transcender los aspectos económico y comercial, desarrollando un tipo de intercambio "cultural y social e incluyendo a la sociedad civil en el proceso de integración", además de los gobiernos ${ }^{8}$.

Además de las iniciativas de gobierno con esa misma óptica, cabe registrar que se dio seguimiento a la directriz de "Divulgar Porto Alegre y sus atracciones" -no sólo en el Cono Sur y en el país- con la producción de material promocional con versión en inglés, tanto sobre el Presupuesto Participativo como sobre los aspectos más generales de la ciudad. Se observa que en la imagen difundida se asocian elementos económicos, ambientales, culturales y políticos: "Porto Alegre, una ciudad que combina cultura, ocio, ecología y desarrollo con tecnología y participación" (Porto Alegre, PMPA, 1996, material promocional).
De este modo, junto a la infraestructura y a los servicios disponibles, se vende también la ciudad como "gestión democrática y participación popular", la ciudad "culturalmente rica y diversificada" y con "cualidades de vida ambiental". Se trata de una imagen multifacética que aparece como ventajas comparativas para convertir la ciudad competitiva en un proceso de integración al mercado mundial.

Por último, se destacó otro aspecto que evidencia cómo rasgos del "emprendedurismo" competitivo asumen matices diferenciados en esa experiencia. El papel del gobierno local como articulador de las fuerzas sociales, a través de formas de cooperación público-privado, se torna más propositivo e inductor. Como destacó el ex-alcalde Tarso Genro: se trata de un gobierno con "capacidad de irradiar nuevos conceptos en las diversas áreas" y un "gestar político de economía”. Eso se traduce, por ejemplo, en una noción de cooperación público-privado que enfatiza la construcción del interés público, siguiendo un referencial democrático. El proyecto Ciudad Constituyente, así como las directrices definidas e implementadas en el área económica, entre otras, reproducen esa óptica, que asocia cooperación público-privado con negociación, participación y disputa democrática de intereses y proyectos. Se asume que el gobierno tiene un papel destacado en la discusión de su(s) proyecto(s) político(s) en los diversos espacios públicos.

En esta asociación, entre el activismo democrático y el "emprendedurismo", no parece haber una mera reproducción de los aspectos de cada uno. Éstos se redefinen, renovando, de cierta forma, el discurso y la práctica. La incorporación en la agenda local de la preocupación por las estrategias de desarrollo económico y por la integración en el mercado mundial se relaciona con la preocupación por el intercambio solidario entre ciudades, la creación de puestos de trabajo y la distribución de la renta. Se articula la democratización del Estado y la creación de nuevas esferas públicas con la búsqueda de acciones compartidas por la sociedad civil.

\section{LA FORMACIÓN DE REDES PÚBLICAS}

Conforme lo señalado, la constitución de espacios de participación y de cooperación entre el Estado y la sociedad civil es un rasgo característico de la experiencia de Porto Alegre. Tales espacios pueden ser analizados como redes".

7. Directrices para Porto Alegre, PMPA/Congreso de la Ciudad, diciembre de 1993

8. La Red Merco-Ciudades se formó en noviembre de 1995 en Asunción de Paraguay y fue precedida por una reunión realizada en junio del mismo año en Porto Alegre. Además de esas dos ciudades integran la Red: Florianópolis, Belo Horizonte, Curitiba, Rio de Janeiro, Brasília, Salvador, Buenos Aires, Rosario, Córdoba, La Plata y Montevideo. Tiene como objetivo "establecer un intercambio de experiencias políticas y administrativas, capaz de potencializar acciones [...] de las ciudades integradas en la red; constituir un canal de participación de los municipios en el proceso de formación del Mercosur, en el sentido de democratizarlo y convertirlo en una efectiva integración de comunidades" (Compromiso de Porto Alegre firmado por los representantes de los gobiernos de las ciudades citadas, 1995).

9. Se distingue, por un lado, el abordaje de redes como expresión de los nuevos arreglos interorganizacionales que emergen en la actualidad, 
Dependiendo del proyecto, las redes asumen un carácter más o menos formal; son más o menos heterogéneas en términos de los segmentos sociales involucrados; asumen funciones de negociación y discusión democrática de intereses, de movilización de recursos y/o legitimación de proyectos.

En este rol se incluyen desde los espacios de participación directa, como las sesiones plenarias regionales y temáticas del Presupuesto Participativo ${ }^{10}$, pasando por espacios de representación delegada más amplios -el Forum del PP y el Congreso de la Ciudad- hasta las comisiones menores formadas por representantes de entidades y/o de los espacios de participación directa -Consejo del Presupuesto Participativo, Coordinación General en el proyecto Ciudad Constituyente, Consejo Administrativo de la Institución Comunitaria de Crédito Porto Sol, Comisión del Porto Alegre Tecnópolis, Consejo de la Incubadora Tecnológica (AIETEC) y demás proyectos económicos-. Estos últimos asumen un funcionamiento más cotidiano, actuando directamente en la gestión, implicando, por tanto, decisión, articulación de recursos y control sobre las respectivas acciones que están involucradas. Tal patrón organizativo se reproduce en diversas áreas y sectores de la administración municipal.

A medida que los proyectos finalizan o se agotan, esas redes se deshacen o son redefinidas, evidenciando un tipo de organización más próxima a los nuevos conceptos de red. Es decir, no son estructuras fijas; existen en cuanto persisten los problemas/motivos y condiciones que las crearon. Por otro lado, tienden a asumir nuevas configuraciones, bien sea por la rotación de los actores, bien por el aprendizaje. Es ilustrativo el caso del Presupuesto Participativo, cuya configuración estructural está asumiendo nuevas formas a lo largo del tiempo, con la inclusión, por ejemplo, de plenarias temáticas paralelas a las regionales.

Los conflictos y las tensiones en esos espacios, sobre todo los más heterogéneos, conviven con la posibilidad de cooperación y negociación. En Ciudad Constituyente, por ejemplo, se observó que, dada la diversidad de los actores participantes y, por tanto, de los intereses en juego, la actitud cooperativa y la formación de alianzas puntuales fueron sobrepasadas por conflictos y por la articulación de bloques entre los segmentos que comportan valores comunes. En cuanto a la naturaleza de estos conflictos, se constató que emergían, principalmente, de la metodología participativa: por un lado, de la tensión entre un abordaje técnico de los problemas y proyectos de desarrollo futuro de la ciudad y la aproximación sectorial de los intereses sociales en juego; por otro, de la dirección del proceso, con sus limitaciones y ambigüedades en el grado de apertura democrática.

La apertura de esas redes para la entrada de nuevos integrantes a lo largo del proceso es otro aspecto interesante y va a depender, entre otros, del atractivo del proyecto, del trabajo de articulación e integración de los actores sociales y de los resultados alcanzados. En Ciudad Constituyente, por ejemplo, se verificó que la diferencia entre el número de invitados y los participantes se debía a problemas de orden metodológico, imprecisiones conceptuales y lagunas en cuanto a la producción y difusión de informaciones, entre otros factores. Al mismo tiempo, la desconfianza generada por la nueva idea que se quería implementar así como la existen cia de un escenario el el cual el gobierno local no detentaba la hegemonía sobre el conjunto de los segmentos sociales, no fueron acompañadas con un trabajo inicial de convencimiento y compromiso con el proyecto ${ }^{11}$. El respaldo del Presupuesto Participativo, por otro lado, medido por la amplitud de la participación en las sesiones plenarias y por el reconocimiento público, tiene mucho que ver con la eficacia del proceso participativo, que aparece con la resolución de demandas a partir de lo acordado colectivamente.

Independientemente de los resultados y limitaciones, la existencia de múltiples espacios públicos de participación/cooperación es valorada por actores de la sociedad civil positivamente, aunque con algunas reservas.

\section{POSIBILIDADES Y LÍMITES}

La experiencia de Porto alegre evidencia tanto factores favorables a la democratización y gestión ciudadana, que deben de profundizarse, como también algunas limitaciones que deben de tomarse en cuenta. En primer lugar, cabe resaltar que la diversificación de las acciones en el campo económico conforme lo ocurrido en Porto Alegre, puede ser un indicador de las potencialidades municipales en este campo. No obstante, se han de considerar los límites estructurales en la medida que decisiones fundamentales que afectan a la economía local pueden ser asumidas por otras esferas del gobierno y por las corporaciones multinacionales.

En segundo lugar, deben de verificarse los instrumentos de los cuales dispone un ayuntamiento para la gestión económica como por ejemplo, los instrumentos de política fiscal clásicos: bajo un enfoque keynesiano, el gobierno municipal se ha valido del gasto público y así ha evitado el uso de los beneficios tributarios, por opción política. Los gastos, en cuanto a inversión y consumo público son los que pueden garantizar la operacionalización de los proyectos económicos lo que presupone la existencia de recursos financieros, la capacidad de inversión de las finanzas públicas locales y de movilización de los recursos externos.

Cabe resaltar que, hasta entonces, en el proceso de discusión pública del Presupuesto Municipal, la temática de desarrollo económico no había figurado entre las siete prio-

indicando el aumento de los procesos de interdependencia entre actores y organizaciones $y$, particularmente, entre agentes públicos y privados Expresan un tipo de articulación que congrega una pluralidad de actores, menos jerárquica y estructurada, en torno a problemas/proyectos delimi tados y basadas en relaciones más o menos formales y regulares. Se destacan, también, las ideas de coooperación, para la movilización de recursos y/o el procesamiento de conflitos y divergencias, la negociación y la demo cratización de los procesos decisorios. Por otro lado, también se utilizan las redes como instrumento de análisis de las características de las confi guraciones y formas de interacción entre actores y organizaciones, de forma genérica. Ver MOURA (1997)

10. Estas sesiones que son informativas/consultivas pueden alcanzar un nivel decisorio sobre las prioridades de la región/temática y la elección de representantes para el Consejo del Presupuesto y el Forum de Delegados.

11. Al contrario de lo sucedido en Barcelona con el uso de la Planificación Estratégica donde el compromiso fue uno de los factores que contribuyó a la entrada y permanencia de los actores considerados estratégicos en el proceso. 
ridades de inversión. En el Plan de Inversión de 1998, las acciones en pro del desarrollo quedaron en octavo lugar, y las propuestas en el área de la ocupación y renta aparecieron como una de las prioridades regionales en, apenas, dos regiones de la ciudad.

En realidad, los recursos destinados a la secretaría responsable del área económica (SMIC) son bastante reducidos: cerca del $1 \%$ del presupuesto total del ayuntamiento. Lo mismo se observa cuando se compara el volumen de recursos captados por la Secretaría de Captación de Recursos (SECAR) para inversión, vía préstamo o a fondo perdido. Para los proyectos de infraestructura y equipamientos urbanos, relacionados a las áreas más tradicionales de actuación del ayuntamiento, fueron recaudados 187.3 millones de reales. Mientras que para los proyectos económicos stricto sensu - en este caso, la Institución Comunitaria de Crédito- se obtuvo apenas 3.3 millones de reales, o sea, el 1,6\% del total de recursos recaudados en cuatro años.

Cabe también la posibilidad de considerar que determinados proyectos requieran pocos recursos financieros del municipio, bien sea porque demanden más tareas de articulación y/o porque son pensados para ser ejecutados en cooperación con organizaciones de la sociedad civil y de otras esferas del gobierno. Así pues, sólo lo que entra en los cofres municipales, como recaudaciones propias, transferencias y préstamos, es computado en el presupuesto, excepto en el caso de los recursos aportados por organizaciones privadas. Consecuentemente, los resultados obtenidos en el análisis de la estructura presupuestaria no deben ser considerados suficientes, cuando el objetivo es evaluar la prioridad de tales proyectos.

Las iniciativas económicas del ayuntamiento privilegian a grupos excluidos (colectores de papel, grupos de mujeres de la periferia, creadores clandestinos de puercos) y al segmento de los pequeños y medios emprendedores. Conforme lo visto, uno de los instrumentos básicos de acción es la distribución de recursos municipales, combinada con la movilización en cooperación.

Por otro lado, la relación que el gobierno mantiene con el empresario medio y grande es más de búsqueda de apoyo y de control, que de incentivo directo. Por ejemplo, para aprobar la instalación de un centro comercial en la ciudad en 1999, se exigió la mejoría de la infraestructura vial y la construcción de casas populares ${ }^{12}$. Inclusive existe un decreto que establece las normas para construír grandes proyectos de desarrollo urbano con el fin de evitar impactos negativos sobre los pequeños y medios empresarios y sobre el medio ambiente ${ }^{13}$. Ese tipo de postura choca con la perspectiva más tradicional de desarrollo, entendido como resultado de atracción de las empresas.

La posición adoptada en Porto Alegre con relación a los grandes proyectos de desarrollo representan ensayos en cuanto implican la creación de instrumentos acordes con los principios de la precaución y del contaminador/usuario pagador, típicos de los modelos actuales de desarrollo sostenido ${ }^{14}$. Pero, incluso con esas experiencias, se ha innovado poco en materia de instrumentos para la gestión local de la economía.

Además de tomar en cuenta los instrumentos regulatorios, debe dirigirse el análisis hacia aquellos factores subje- tivos que influencian la gestión local del desarrollo en Porto Alegre. Se observa, por un lado, que la continuidad política, garantizada por dos reelecciones sucesivas y por un cierto grado de cohesión partidaria ${ }^{15}$, aparece como un factor que permite la profundización y el aprendizaje necesarios para el desarrollo de proyectos que se pretenden innovadores. Tal continuidad, al mismo tiempo, no ha implicado homogeneidad. Al contrario, existen diferencias debido a la expresión de distintas tendencias en el gobierno local, dentro del mismo conjunto de fuerzas, y a las particularidades de los líderes. La expresión de esas diferencias, en un cuadro de continuidad, ha posibilitado la experimentación y el enriquecimiento de las acciones estratégicas.

Otro factor que ha contribuido a impulsar esa experiencia está relacionado con el grado de organización de la sociedad civil. En el campo de los sectores populares se ha de resaltar la trayectoria de los movimientos populares urbanos, (que se remontan a los años 70/80), en torno a la problemática local de vivienda: las villas miseria. Tales movimientos constituyeron asociaciones de vecinos y similares, articuladas en las regiones más pobres de la ciudad ${ }^{16}$. Este patrón organizativo -las denominadas Uniones de villas y, posteriormente, los Consejos Populares-sirvieron de base, por ejemplo, para la estructuración del Presupuesto Participativo. Cabe registrar la presencia de algunas $\mathrm{ONGs}^{17}$ actuando en esos movimientos que asumen, también, un papel de articuladores en las redes públicas y en la constitución de otros foros, como el de la Reforma Urbana ${ }^{18}$.

12. Dichas casas estaban destinadas al realojamiento de familias que vivían en situación de "ocupación" en los alrededores.

13. Los desarrollos con un área superior a 2000 metros cuadrados, están obligados a proveer un relatorio de impacto ambiental y otro de posibles impactos sobre el mercado en relación a las micro, pequeñas y medias empresas, además de comprometerse con medidas compensatorias de los efectos negativos. (Porto Alegre Agora, PMPA, junio/98, Año X, N. 46, pag. 9).

14. Esos principios son ampliamente discutidos por los teóricos del desarrollo ambiental sostenido. En los países desarrollados, instrumentos de política gubernamental inspirados en ellos ya son de uso racional. Aunque sean preferencialmente discutidos y analizados en el ámbito de la literatura sobre medio ambiente y sustentabilidad, los autores los consideran pertinentes a proyectos de desarrollo local sostenido

15. El P'T en Rio Grande del Sur, en el ámbito del partido, siempre se destacó como un modelo de estructuración y de convivencia democrática de las diferentes tendencias existentes en su interior. Esto remite, entre otros factores, a la propia historia/cultura política del Estado, donde los partidos son comparativamente más estructurados que en el resto del país, constituyéndose en espacios de cohesión y representación política.

16. A mediados de los 80 se creó una federación municipal de asociaciones de vecinos, la UAMPA, que procuró constituirse como espacio de articulación y de impulso del movimiento popular en la ciudad. Con todo, encontró dificultades y no se ha destacado como actor relevante en los procesos participativos, como el PP.

17. La más antigua de las ONGs, creada a final de los 60 , forma parte de una organización de ámbito nacional -a FASE-. En función de la reestructuración por la que pasó la entidad después de los 90, la oficina de Porto Alegre se cerró en 1996. Hasta el momento de finalización de la investigación, se constató la presencia de otras dos con actuación en los movimientos y organizaciones populares urbanas -el CIDADE (Centro de Estudios y Asesoría Urbana) y el CAMP (Centro de Asesoría Multiprofesional)-.

18. El Forum Municipal de Reforma Urbana fue creado en julio de 1994, en un contexto de desintegración de lo que hasta entonces se había constituido en el ámbito estatal, cuando estaba en pauta en Porto Alegre la política habitacional y de regularización fundiaria. Está coordinado por ONGs y por un representante del Consejo del OP, con reuniones mensuales que congregan un público diverso, como la universidad y líderes de base. 
Las organizaciones sindicales de trabajadores, inclusive las centrales de la CUT (Central Única de Trabajadores) y de la CGT (Central General de los Trabajadores), aunque están presentes en el escenario estatal, no tienen una actuación decisiva en cuestiones relacionadas con las políticas municipales, hecho común en Brasil ${ }^{19}$. Algunas iniciativas, sin embargo, fueron tomadas desde el gobierno de Olivio Dutra en el sentido de articularlas. Algo que se explica por los vínculos orgánicos del PT con el movimiento y en función de representar ese campo de organización popular en el programa partidario. Se destaca la creación en 1989 del Forum Sindical, que "se reúne una vez por mes para discutir problemas y políticas de interés sindical que puedan ser encaminadas por el ayuntamiento". Pero recientemente, con las iniciativas económicas del ayuntamiento y considerando el aumento de desempleo, hay un espacio más propicio para la actuación sindical. El discurso del director de la CUT ilustra estas observaciones:

"La CUT fue tomando conciencia de que era necesario disputar la construcción de la política de la ciudad. Además del Forum Sindical, existen otros, el de la Secretaria Municipal de Producción, Industria y Comercio, sobre política industrial, el Porto Alegre Tecnópolis [...] El Presupuesto Participativo con las sesiones plenarias temáticas hizo más adecuada la participación sindical, así como Ciudad Constituyente, que encierra una discusión más política de visión para el futuro de la ciudad" (Pedro Correia, CUT Metropolitana, entrevistado el 02/10/96).

Entre los sectores medios, cabe mencionar la presencia de las asociaciones profesionales, como la Asociación de Talleres de Arquitectura y la Sociedad de Economía, además de las organizaciones que agregan profesionales del área artística y cultural, por ejemplo la Asociación de Artesanos. Tales organizaciones también asumen importancia en los procesos participativos y/o en la cooperación que está en la base de los proyectos de desarrollo local, siguiendo la lógica de implicación en cuestiones de interés de las categorías. Por ejemplo, los arquitectos en el proceso de reformulación del Plan Director, como desdoblamiento de la Ciudad Constituyente, y los artesanos y artistas con relación al proyecto del Corredor Cultural, de dinamización del centro histórico.

$\mathrm{La}$ existencia de asociaciones de interés que integran diversos segmentos sociales no impide que el gobierno encuentre dificultades para llevar adelante proyectos participativos. Por un lado, se observa que, no siempre los espacios abiertos se vuelven atractivos, bien porque no despiertan interés, o porque existen desconfianzas y resistencias político-ideológicas. Tal dificultad fue encontrada, por ejemplo, en Ciudad Constituyente, donde de las diecisiete organizaciones invitadas para integrar la coordinación general, solo ocho participaron. Por otro lado, no siempre la integración a los espacios constituidos implica un posicionamiento participativo y propositivo de las organizaciones de la sociedad civil.

Al mismo tiempo, es interesante observar que la representación de asociaciones en los espacios públicos convive, en muchos casos, con la dificultad de integración de las bases en el proceso. Hecho ya analizado por la literatura sobre el tema y, también, observado en varias de las declaraciones, como la siguiente:

\begin{abstract}
"Me enfrenté al problema, como representante de la universidad, de ¿cómo hacer fluir la comunicación? Con quién debería contactar, sin espacio ni momentos de confluencia colectiva? Estaba representando al gabinete del rector o a la universidad como un todo?" (Zilá Mesquita, UFRGS. Entrevistada el 27/09/96).

"Existe una dificultad del movimiento sindical para discutir con sus asociados. No se hicieron asambleas, hubo más participación de las direcciones. Encontrábamos compañeros participando a través del movimiento popular (de barrio)". (Pedro Correia, CUT Metropolitana, entrevistado el 02/10/96).
\end{abstract}

La situación varía cuando hay capacidad de articulación de los líderes aliada al grado de interés que los procesos en cuestión despiertan. Ese es el caso del Presupuesto Participativo que implica tomar decisiones sobre la aplicación de recursos públicos y generar espacios amplios tales como las sesiones plenarias, las cuales tienen competencia para elegir representantes para el consejo municipal quien, a su vez tiene el poder para tomar las decisiones anteriores. El hecho de que parcelas importantes de la sociedad no estén organizadas es un factor restrictivo para la implementación de proyectos económicos y de otro tipo que requieren un alto nivel de articulación entre el ayuntamiento y el público-objeto del mismo. Otro aspecto que debe de destacarse como limitante para un posicionamiento más participativo en los proyectos estratégicos, se refiere a la presencia del conservadurismo entre los agentes económicos especialmente cuando se trata de experimentar con nuevas experiencias que desafían posiciones cristalizadas y que envuelven intereses económicos.

Existen limitaciones para la realización de los objetivos estratégicos no sólo en el campo de la sociedad civil, sino también en el interior del proprio gobierno y de la administración municipal, remitiendo a dificultades de índole diversa. El posicionamiento ambiguo de los dirigentes del gobierno en espacios públicos, de apertura y cierre a la participación de los actores sociales, es un factor que limita la interferencia social en los procesos, volviendo la participación más formal que substantiva en algunos casos. En estos casos, los espacios públicos sirven más para homologar y legitimar decisiones tomadas en otras instancias que para definir y acompañar la gestión de proyectos.

Observaciones como estas, hechas en relación a una experiencia, ciertamente no pueden ser generalizadas. Pero

19. El movimiento sindical en Brasil se ha dedicado fundamentalmente a cuestiones relacionadas al salario o a condiciones de trabajo, o sea, mantiene su actuación de "la puerta de la fábrica para dentro". Esa es una diferencia con relación a la experiencia de la Unión Sindical Comisiones Obreras de Barcelona, que montó una estructura específica para tratar cuestiones del "lado de fuera de la fábrica" (como transporte, vivienda, calidad de vida, etc.) Por eso se justifica la participación destacada de esta central en el proceso de planificación estratégica. 
pueden ser consideradas como expresiones de una tendencia. Tendencia que remite a la propia dinámica interna de los aparatos del Estado, estructurados bajo bases burocráticas y jerárquicas, siguiendo por tanto una lógica distinta de los procesos participativos.

La implementación de proyectos que implican movilización, cooperación y participación de la ciudadanía, requiere no sólo la existencia de un tejido asociativo dinámico y voluntad política, sino también un buen esquema de producción y difusión de informaciones y la construcción de metodologías adecuadas. Se observan algunas limitaciones en estos dos campos.

En el estudio sobre Ciudad Constituyente aparecen insuficiencias en la divulgación para la movilización e inadecuaciones en la producción de informaciones accesibles a los actores que participan. El tipo de metodología adoptada también repercute en la dinámica de los procesos y puede convertirse en un factor restrictivo. Existen problemas como la inadecuación del horario de funcionamiento de las reuniones hasta problemas de estructuración de espacios participativos que combinen debates ampliados con estudios, formulación y definición de políticas. A título de ilustración cabe rescatar algunos problemas identificados en Ciudad Constituyente. Con los grupos de trabajo se pretendía llegar a un nivel de formulación de estrategias y directrices de desarrollo, siendo el punto de partida la realización de un diagnóstico de la realidad local. En la práctica, los grupos sirvieron como espacio de agregación de algunos estudios sectoriales ya realizados, dentro y fuera de la administración municipal, y de demandas y propuestas con niveles variados de formulación, destacándose lo que venía siendo producido por sectores del gobierno más directamente involucrados en el proceso ${ }^{20}$. Pero no llegaron a realizar estudios. Formularon síntesis sobre problemáticas de la realidad local, directrices del gobierno - basadas en el trabajo de los coordinadores de los GTs - y, en total, cerca de 300 recomendaciones, variando de declaración de principios a sugerencias de programas y proyectos.

La evaluación sobre el no funcionamiento de los GTs como grupo de trabajo puede atribuirse a un problema de concepción metodológica del proyecto. $\mathrm{O}$ sea, se hace difícil realizar estudios y formular estrategias y directrices generales de desarrollo a partir de grupos abiertos a la participación de los diversos segmentos sociales, con una dinámica de reuniones semanales por la noche y, también, con insuficiencia de datos sistematizados sobre la realidad local. Puesto que entre los diversos actores invitados a participar en los procesos existe una tendencia a la identificación de temas específicos en virtud de sus intereses y demandas, es preciso preguntarse si en los casos en que se dispone de estudios y se hacen propuestas y proyectos con cierto nivel de elaboración, no se debería incorporar tro tipo de metodología de trabajo que tome en consideración los objetivos propuestos ${ }^{21}$.

Con este ejemplo se evidencia la complejidad de la estructuración de procesos participativos, que está en la base de los proyectos estratégicos en Porto Alegre y se convierte en un tema clave para el éxito de los mismos. Por eso es fundamental el aprendizaje y una postura adaptativa. En este sentido es interesante la declaración de un líder del barrio:
"Todo el mundo está desactualizado en las nuevas realidades que están ahí. Tiene que haber continuidad de esas discusiones públicas, abiertas...Todo el mundo tiene que estudiar más: los de la comunidad porque no saben algunas cosas, los técnicos porque no saben lidiar con las comunidades". (Neusa Amoretti, Consejo del Presupuesto Participativo, entrevistada el 11/09/96).

Inclusive con las limitaciones señaladas, ese aspecto del aprendizaje puede ser considerado como un factor que potencializa la experiencia.

"Aunque el proceso se haya dado con peleas y la gente se sienta medio sin conocimiento necesario sobre como planificar de una forma nueva, aportó novedad. Al participar, [...] mucha gente procuró profundizar, pero esa profundización exige más tiempo, más conocimiento sobre como ir [...] hubo esfuerzos en ese sentido, como el documento preparado por el personal del Presupuesto Participativo [...] también las ONGs, los técnicos del ayuntamiento, están aprendiendo. Todavía se está aprendiendo lo que es planificación estratégica. Lo nuevo tiene que ser construido" (Regina Pozzobon y Leticia Marques, CIDADE, entrevistadas el 02/10/96)

\section{CONSIDERACIONES FINALES}

La construcción de una estrategia de desarrollo local en Porto Alegre, comparada con otras experiencias que están siendo difundidas como innovadoras, sigue una lógica muy particular. Se diferencia, por ejemplo, del modelo de planificación estratégica de Barcelona, adoptado en ciudades brasileñas, como en Río de Janeiro y Vitoria. Conforme lo analizado, no se observa una estructura ni un momento específico de elaboración de un plan, sino la conformación de una serie de acciones/programas orientados por una visión estratégica, explícitamente político-partidaria, que se construye y se profundiza durante tres gobiernos sucesivos. El proceso es semejante al de Curitiba, otra ciudad brasileña, aunque aquí la orientación y los actores propulsores no se encuentran claramente identificados con un proyecto partidario y el contenido de las acciones obedece más a una lógica de búsqueda de la eficiencia de la ciudad y de la gestión urbana, para hacerla más competitiva.

20. Los casos de la Secretaría Municipal de Producción, Industria y Comercio, con relación a la temática de desarrollo económico; de la Secretaría Municipal de Transporte, en cuanto a circulación y transporte; de la Secretaria Municipal de Hacienda, con la discusión del financiamiento de la ciudad; de la Secretaría de Planificación Municipal, con aspectos de la discusión del desarrollo urbano. Se destacan también estudios y propuestas de otros actores, como la de los investigadores de la Universidad Federal de Rio Grande del Sur, de la Fundación de Economía y Estadística del gobierno del estado.

21. La solución que se encontró en el segundo momento de Ciudad Constituyente fue la creación de grupos fijos compuestos básicamente por técnicos con una dinámica más cotidiana de trabajo que deberían funcionar paralelamente a los grupos ampliados con una dinámica de discusiones sobre lo producido por los primeros. 
Lo que puede ser considerado como innovador en esta experiencia es la posibilidad de ampliación de la actuación del gobierno local en dirección a la economía, manteniéndose como referencia el ideario democrático de gestión pública el cual se renueva incluso con la incorporación de rasgos de "emprendedurismo" competitivo. En este proceso no parece haber una mera reproducción de los términos de una y otra tendencia; por el contrario, se redefinen, renovándose en cierta forma, el discurso y la práctica. Por un lado, la inserción en la agenda local de la preocupación con el desarrollo económico y con la integración en el mercado mundial se relaciona con la lucha a la exclusión social,a la distribución de la renta y al intercambio solidario entre ciudades; por otro lado, la búsqueda de acciones compartidas con la sociedad civil se articula con la problemática de la democratización del Estado y la construcción de nuevas esferas públicas.

Entre las limitaciones a este proceso de innovación se pueden destacar las siguientes:

- La economía local fuertemente influenciada por decisiones tomadas tanto en otras esferas de gobierno como por corporaciones multinacionales.

- El bajo grado de organización y dinamismo de la sociedad civil.

- La existencia de una cultura política y administrativa con tendencia autoritaria y burocrática.

- La inadecuación de los mecanismos de producción y difusión de informaciones.

- La fragilidad de los referentes teórico-metodológicos.

Una última reflexión sobre los instrumentos de los cuales puede disponer el gobierno local para la gestión de la economía tiene que ver con la tributación. La progresividad y las exenciones, pueden ser utilizadas en el ámbito municipal, en relación a los impuestos sobre servicios, sobre la propiedad y sobre la transmisión de bienes inmobiliarios. Existen casos, como el de Porto Alegre, en que esos instrumentos no están siendo utilizados, por opción política, para fines de política económica -en el sentido de atraer o incentivar actividades económicas-. Si bien se producen impactos negativos para las finanzas municipales y, particularmente, sobre la capacidad de inversión pública, su utilización podría ser repensada, aunque sea en el marco de un modelo de gestión local que procure superar el modelo tradicional de oferta de beneficios públicos de modo paternalista.

En lo que se refiere a los gastos, el uso dado a los recursos puede ir más allá de la infraestructura y de los servicios urbanos más tradicionales. En el ámbito municipal es posible pensar en la utilización de otros mecanismos de inducción y estímulo a las actividades económicas tales como la oferta de crédito en condiciones más favorables que en el mercado, la producción y/o difusión de tecnologías y la capacitación gerencial y técnica. Los costos públicos de pequeños proyectos de desarrollo tienden a ser relativamente menores cuando se ven como un incentivo a los grandes proyectos de desarrollo. Primero, por una cuestión de escala de inversión. Segundo, porque se puede desarrollar una política que articule y movilice recursos privados y de otras esferas de gobierno.
Sin duda no se puede dejar de considerar la disponibilidad de recursos como uno de los factores limitadores. Tal disponibilidad guarda relación, por un lado, con la situación de las finanzas locales y la capacidad de movilización de recursos externos y, por otro, con el lugar que ocupa el tema del desarrollo económico local en la agenda pública municipal, o sea, cuál es la escala de prioridad.

En relación al aspecto de movilización de recursos externos al ayuntamiento, el municipio puede articular actividades de cooperación con organizaciones privadas y con otras esferas del gobierno para así captar préstamos, junto a organismos de financiamiento nacionales e internacionales. Tales préstamos dependen del aval del gobierno federal, siendo uno de los parámetros analizados la capacidad de endeudamiento del municipio y, por tanto, la situación de las cuentas municipales. También existe la vía de donación por parte de agencias de fomento internacionales y no gubernamentales.

Además de los instrumentos citados, se observa la utilización de prerrogativas municipales en materia de control y orientación de las actividades económicas. El ejemplo de la legislación sobre localización y mega-desarrollos en Porto Alegre indica la posibilidad del municipio de influir sobre el tipo de inversión privada que se considere más adecuada a la estrategia de desarrollo. En esa línea se sitúan, también, la limitación en la localización de industrias contaminadoras, la exigencia de realización de estudios de impacto ambiental y socio-económico y la realización de acciones previas que corrijan impactos negativos.

Además de los instrumentos para la gestión local de desarrollo, otras cuestiones merecen ser profundizadas en estudios posteriores. ¿Cuáles son los efectos multiplicadores de las iniciativas económicas locales? ¿En qué medida tales iniciativas económicas implican crecimiento de la economía popular y/o cuanto correspondería a una mera redistribución de la riqueza ya existente?

\section{BIBLIOGRAFÍA}

Batley, R. (1991): “Comparisons and Lessons". In Batley, R. et STOKER, G. (Org.) Local Government in Europe: Trends and Devellopments. Londres: Macmillan Education Ltda.

Biarez, S. y Nevers, J. (orgs) (1994): Gouvernement Local et Politique Urbaines - Actes du Colloque International. Grenoble: CERAT.

BORJA, Jordi (1994 -a-): Las Ciudades y el Planeamiento Estrategico: una reflexion Europea e Latinoamericana. Barcelona: $\sin$ publicar

CaCcia-Bava, Silvio (1994): "Trocando a Riqueza de Mãos: Distribuição de Renda e Poder nas Pequenas Cidades”. En Revista Proposta, n. 54, p.12-16. Rio de Janeiro: FASE.

DAnIE, Celso (1990): “As Administrações Democráticas e Populares em Questão”. En Espaço e Debates n.30, p. 11-27. São Paulo: NERU

- (1994): Gestão Local e Participação da Sociedade. En VillasBOAS, R. (Org) Participação Popular nos Governos Locais. São Paulo: POLIS (POLIS Publicações, n.14).

FEDOZZI, Luciano (1998): Orcamento Participativo. Reflexoes sobre a Experiencia de Porto Alegre. Rio de Janeiro: Tomo Editorial/ FASE.

Giacomoni, J. (1994): A Comunidade como Instância Executora do Orçamento: a Experiência do Orçamento Participativo de Porto 
Alegre. Dissertação (Mestrado em Administração), PPGA/ UFRGS.

HARVEY, David (1989): "From Managerialism to Entrepreneurialism: The Transformation in Urban Governance in Late Capitalism”. En Geografiska Annaler, n.71b, p. 3-17. Estocolmo. KowARICK, Lucio e Singer, André (1993): “A Experiência do Partido dos Trabalhadores na Prefeitura de São Paulo". Novos Estudos CEBRAP, n.35, p.195-216, marzo. São Paulo.

LEAL, Suely (1994): Para além do Estado: tenências, limites e alcance das novas formas de Gestão Urbana. Tese (Doutorado em Economia). Campinas: UNICAMP.

LE GALÈs, P. (1995): "Du Gouvernement des Villes à la Gouvernance Urbaine”. En Revue Française de Science Politique, v.45, n.1, p. 57-95, fev. Paris: Fondation Nacionale de Sciences Politiques.

- (1994): "Villes en Compétition?". En Biarez, S. y Nevers, J. (orgs): Gouvernement Local et Politiques Urbaines - Actes du Colloque International, Grenoble, CERAT.

Moura, Suzana (1997): Cidades Emprendedoras, Cidades Democráticas e Redes Públicas: Tendências à Renovação na Gestão Local. Tese (Doutorado em Administração Pública), NPGA/EAUFBA. Salvador.
- (1993): "Governo Local e Participação Popular: Ideário e Prática”. En Panizzi, Wrana y Rovatti, João (orgs). En: Estudos Urbanos: Porto Alegre e seus Planejamentos. Porto Alegre, Editora Universidade/Prefeitura Municipal.

- (1993): "Inovações Municipais em Tempos de Democratização e Crise: Novos Cenários da Gestão Urbana”. En Análise E Dados, n. 1, p. 127-134, jun. Salvador: CEI.

- (1994): "A Questão Publico-Privado: Antigos e Novos Sentidos". XVIII ENANPAD, Curitiba. In: Anais... Curitiba: ANPAD.

OSBORNE, David y GAEBLER, Ted (1993): Reinventing Government: How the Entrepeneurial Spirit is Transforming the Public Sector. New York: A Plume Book.

PACHECO, Regina (1993): Iniciativa Econômica Local: a Experiência do ABC. En: Lodovici, E. Samek y Bernareggi, G. M (org). Parceria Publico-Privado: Cooperação Financeira e Organizacional entre o Setor Privado e Administrações Públicas Locais v. 2. São Paulo: Summus.

Pinhanez (1993): et al. Curitiba, uma Estratégia de Marketing. São Paulo, EAESP/FGV, sin publicar.

SiLvA, Tarcilla L. (1993): da et alli. Governo Local Empreendedor em Curitiba: Mito ou Realidade? São Paulo: EAESP/FGV, sin publicar.

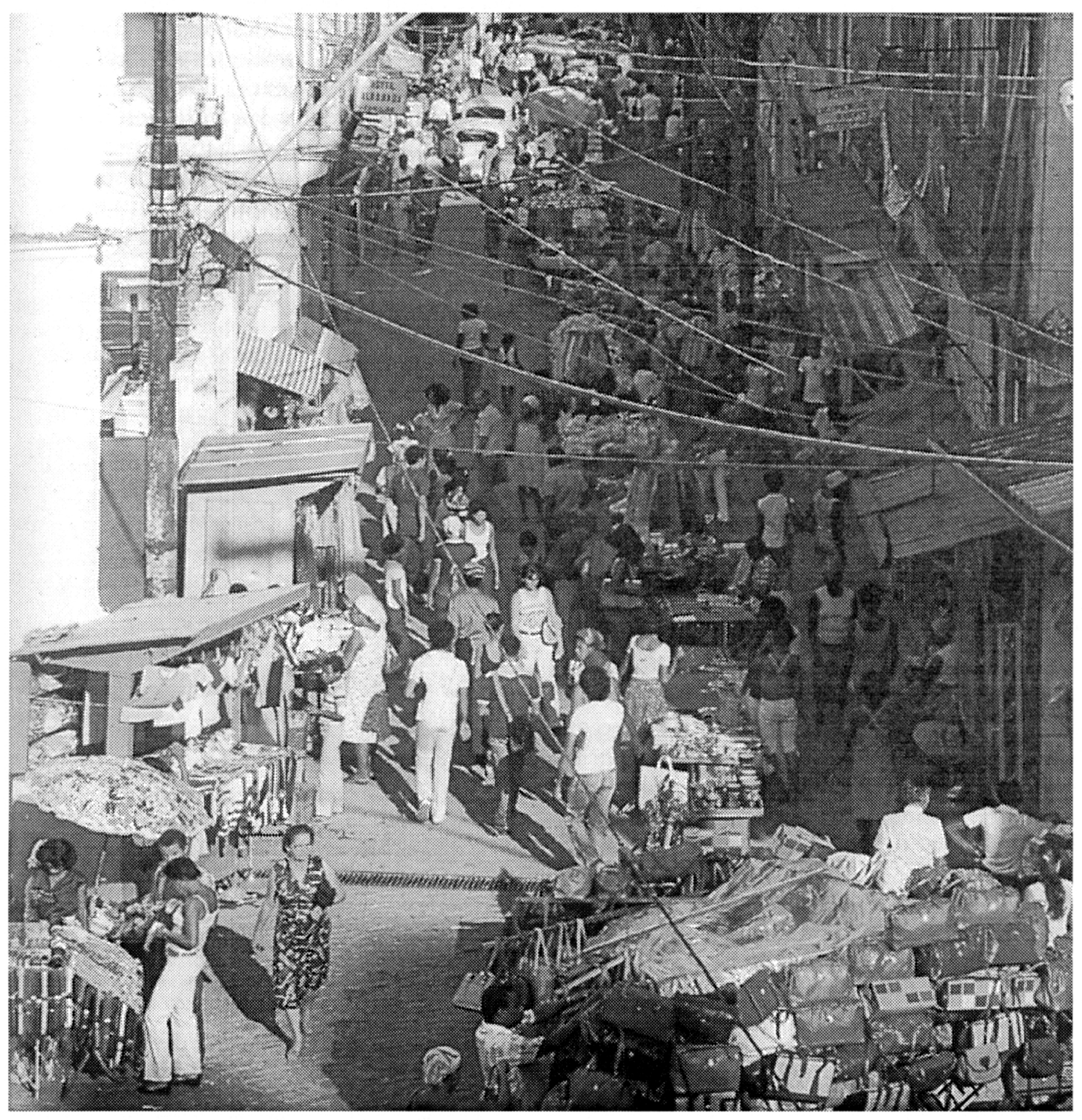

